\title{
Onchocerca parasites and Wolbachia endosymbionts: evaluation of a spectrum of antibiotic types for activity against Onchocerca
} gutturosa in vitro

\author{
Simon Townson*1, Senyo Tagboto ${ }^{1}$, Helen F McGarry², Gillian L Egerton ${ }^{2}$
} and Mark J Taylor ${ }^{2}$

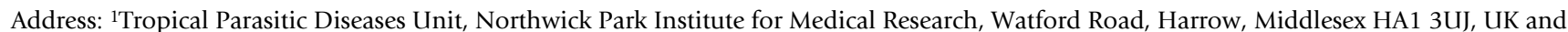
2Filariasis Research Laboratory, Molecular and Biochemical Parasitology, Liverpool School of Tropical Medicine, Pembroke Place, Liverpool L3 5QA, UK

Email: Simon Townson* - s.townson@imperial.ac.uk; Senyo Tagboto - senyo.tagboto@uhns.nhs.uk;

Helen F McGarry - Helen.McGarry@hse.gsi.gov.uk; Gillian L Egerton - gillegerton@msn.com; Mark J Taylor - mark.taylor@liv.ac.uk

* Corresponding author
\end{abstract}

Published: 24 March 2006

Filaria Journal 2006, 5:4 doi:10.1186/1475-2883-5-4

This article is available from: http://www.filariajournal.com/content/5///4

(c) 2006 Townson et al; licensee BioMed Central Ltd.

This is an Open Access article distributed under the terms of the Creative Commons Attribution License (http://creativecommons.org/licenses/by/2.0), which permits unrestricted use, distribution, and reproduction in any medium, provided the original work is properly cited.
Received: 09 February 2006

Accepted: 24 March 2006

\begin{abstract}
Background: The filarial parasites of major importance in humans contain the symbiotic bacterium Wolbachia and recent studies have shown that targeting of these bacteria with antibiotics results in a reduction in worm viability, development, embryogenesis, and survival. Doxycycline has been effective in human trials, but there is a need to develop drugs that can be given for shorter periods and to pregnant women and children. The World Health Organisation-approved assay to screen for anti-filarial activity in vitro uses male Onchocerca gutturosa, with effects being determined by worm motility and viability as measured by reduction of MTT to MTT formazan. Here we have used this system to screen antibiotics for anti-filarial activity. In addition we have determined the contribution of Wolbachia depletion to the MTT reduction assay.
\end{abstract}

Methods: Adult male $O$. gutturosa were cultured on a monkey kidney cell (LLCMK 2) feeder layer in 24-well plates with antibiotics and antibiotic combinations (6 to 10 worms per group). The macrofilaricide CGP 6140 (Amocarzine) was used as a positive control. Worm viability was assessed by two methods, (i) motility levels and (ii) MTT/formazan colorimetry. Worm motility was scored on a scale of 0 (immotile) to 10 (maximum) every 5 days up to 40 days. On day 40 worm viability was evaluated by MTT/formazan colorimetry, and results were expressed as a mean percentage reduction compared with untreated control values at day 40. To determine the contribution of Wolbachia to the MTT assay, the MTT formazan formation of an insect cell-line (C6) 36) with or without insect Wolbachia infection and treated or untreated with tetracycline was compared.

Results: Antibiotics with known anti-Wolbachia activity were efficacious in this system. Rifampicin $\left(5 \times 10^{-5} \mathrm{M}\right)$ was the most effective anti-mycobacterial agent; clofazimine $\left(1.25 \times 10^{-5} \mathrm{M}\right.$ and $3.13 \times$ $10-6 \mathrm{M})$ produced a gradual reduction in motility and by 40 days had reduced worm viability. The other anti-mycobacterial drugs tested had limited or no activity. Doxycycline $\left(5 \times 10^{-5} \mathrm{M}\right)$ was filaricidal, but minocycline was more effective and at a lower concentration $\left(5 \times 10^{-5} \mathrm{M}\right.$ and $1.25 \times$ 10-5M). Inactive compounds included erythromycin, oxytetracycline, trimethoprim and 
sulphamethoxazole. The MTT assay on the insect cell-line showed that Wolbachia made a significant contribution to the metabolic activity within the cells, which could be reduced when they were exposed to tetracycline.

Conclusion: The $O$. gutturosa adult male screen for anti-filarial drug activity is also valid for the screening of antibiotics for anti-Wolbachia activity. In agreement with previous findings, rifampicin and doxycycline were effective; however, the most active antibiotic was minocycline. Wolbachia contributed to the formation of MTT formazan in the MTT assay of viability and is therefore not exclusively a measure of worm viability and indicates that Wolbachia contributes directly to the metabolic activity of the nematode.

\section{Background}

The control of human filariasis caused by Brugia malayi, Wuchereria bancrofti and Onchocerca volvulus, currently relies on community-wide mass distribution of ivermectin and albendazole, either individually or in combination with diethylcarbamazine. Unfortunately, these drugs are mainly microfilaricidal rather than macrofilaricidal, which means that repeated treatment is required over many years, and the possibility that resistance to them may develop is a cause for concern [1-3]. However, with the realisation that many filarial nematodes, including those responsible for most of the global burden of human filarial disease, are infected with Wolbachia [4], has provided an alternative approach to the treatment and control of these parasites with antibiotics.

Wolbachia are intracellular bacteria that have a mutualistic relationship with their nematode hosts [4] and studies have shown that antibiotics can eliminate the bacteria and result in worm growth retardation, infertility and reduced viability [5-8]; conversely, nematodes not infected with Wolbachia were unaffected by antibiotic exposure [5]. Recent field trials of the antibiotics oxytetracycline against onchocerciasis in cattle [9] and of doxycycline in human onchocerciasis [10-12] and W. bancrofti infection $[13,14]$ have demonstrated the validity of this approach. In all cases, treatment resulted in a pronounced reduction in microfilaraemia/microfilaridermia and a prolonged inhibition of embryogenesis, but, significantly, an eight week course of doxycycline $200 \mathrm{mg}$ daily also proved to be macrofilaricidal against $W$. bancrofti [13]. The administration of antibiotics may also have benefits in addition to their sterilising effects and killing of worms; Wolbachia are associated with the pathology of filarial infections [15-17] and with adverse reactions to diethylcarbamazine and ivermectin $[18,19]$. A recent study has shown that a three week course of doxycycline can lead to a sustained amicrofilaraemia but does not result in macrofilaricidal activity [20].

Although antibiotics have a valuable activity against filarial nematodes, the long treatment regimens that are required present logistical problems for mass drug admin- istration (MDA). Another obstacle to MDA is the contraindication of tetracyclines in pregnant women and children under the age of eight years. Therefore, alternative treatment options that target Wolbachia but circumvent these problems would be advantageous. For more than 18 years, an in vitro drug screen for identifying potential macrofilaricidal activity has used adult male O. gutturosa [2123], with assessment of efficacy being made by observing worm motility and inhibition of MTT formazan formation $[24,25]$. In the present work we have extended this system and developed a long-term assay, which has enabled us to screen many of the commonly used antibiotics for macrofilaricidal activity, both individually and in combinations. We have also determined the contribution of Wolbachia to the MTT assay.

\section{Methods \\ In vitro drug screen}

Adult male O. gutturosa were dissected from the nuchal ligament connective tissues obtained from naturally infected cattle in Kumasi, Ghana, as previously described [6]. They were maintained individually in the wells of a 24-well plate containing $1.8 \mathrm{ml}$ of Minimum Essential Medium with $10 \%$ heat inactivated calf serum and a monkey kidney cell (LLCMK2) feeder layer [26] at $36.5^{\circ} \mathrm{C}$ with $5 \% \mathrm{CO}_{2}$ for 24 to 48 hours until the addition of drugs. The medium of all wells included the antibiotics penicillin (200 U/ml) and streptomycin $(200 \mu \mathrm{g} / \mathrm{ml})$, which have no anti-Wolbachia activity [27], and the anti-mycotic agent amphotericin B $(50 \mu \mathrm{g} / \mathrm{ml})$. Drugs were prepared as previously described [6] in medium and each drug concentration was tested against six to ten worms, maintained as above. Medium (with or without drug) was replaced every 5 days. The compounds tested included a range of test antibiotics (Tables 1, 2 and 3), both individually and in combination. The amoscanate derivative CGP 6140 (Amocarzine) was used as a positive control since it is macrofilaricidal against Onchocerca parasites [28], reviewed by [29].

Worm viability was measured by the motility levels and MTT colorimetry. Motility scores were assessed on an inverted microscope on a scale of 0 (immotile) to 10 
Table I: Summary of long-term trial I: parasite mean motility scores and viability (MTT colorimetry)

\begin{tabular}{|c|c|c|c|c|c|c|c|c|c|c|c|}
\hline \multirow{2}{*}{$\begin{array}{l}\text { Compound/drug and conc. } \\
\text { DAY }\end{array}$} & \multicolumn{9}{|c|}{ Mean motility scores over a period of $\mathbf{4 0}$ days } & \multirow{2}{*}{\begin{tabular}{|l} 
Motility \\
\% reduction on \\
day $40(P)$
\end{tabular}} & \multirow{2}{*}{$\begin{array}{c}\text { MTT } \\
\begin{array}{l}\text { inhibition on } \\
\text { day } 40(P)\end{array}\end{array}$} \\
\hline & 1 & 5 & 10 & 15 & 20 & 25 & 30 & 35 & 40 & & \\
\hline Control & 8.0 & 7.4 & 7.1 & 7.0 & 5.3 & 5.5 & 6.3 & 6.4 & 4.9 & & \\
\hline CGP $6 \mid 40 \mathrm{I} .25 \times 10-5 \mathrm{M}$ (positive control) & 0.5 & 0.8 & 0.0 & 0.5 & 0.0 & 0.0 & 0.0 & 0.0 & 0.0 & $100.0(0.005)$ & $83.3(0.098)$ \\
\hline Rifampicin $5 \times 10^{-5} \mathrm{M}$ & 7.7 & 6.8 & 6.7 & 6.8 & 3.2 & 1.7 & 0.2 & 0.2 & 0.0 & $100.0(0.002)$ & $89.6(0.033)$ \\
\hline Rifampicin $1.25 \times 10^{-5} \mathrm{M}$ & 7.0 & 7.0 & 7.3 & 5.7 & 6.5 & 5.2 & 5.7 & 5.0 & 5.6 & $0.0(0.647)$ & $0.0(0.798)$ \\
\hline Minocycline $5 \times 10^{-5} \mathrm{M}$ & 8.0 & 6.7 & 7.3 & 5.7 & 0.0 & 0.0 & 0.0 & 0.0 & 0.0 & $100.0(0.002)$ & $84.1(0.042)$ \\
\hline Minocycline $1.25 \times 10^{-5} \mathrm{M}$ & 7.8 & 7.5 & 6.7 & 5.7 & 4.3 & 2.5 & 4.8 & 1.7 & 0.0 & $100.0(0.002)$ & $93.7(0.026)$ \\
\hline Doxycycline $5 \times 10^{-5} \mathrm{M}$ & 8.3 & 8.0 & 8.0 & 5.7 & 0.3 & 0.0 & 0.0 & 0.0 & 0.0 & $100.0(0.002)$ & $93.0(0.027)$ \\
\hline Doxycycline $1.25 \times 10^{-5} \mathrm{M}$ & 7.3 & 6.5 & 6.3 & 4.8 & 6.3 & 6.3 & 6.2 & 6.2 & 5.5 & $0.0(0.699)$ & $0.0(0.801)$ \\
\hline Ethambutol $5 \times 10^{-5} \mathrm{M}$ & 6.8 & 6.8 & 6.5 & 6.7 & 4.0 & 4.8 & 4.3 & 5.0 & 2.7 & $44.9(0.170)$ & $73.0(0.108)$ \\
\hline Dapsone $5 \times 10^{-5} \mathrm{M}$ & 7.3 & 7.3 & 7.7 & 5.0 & 6.8 & 7.0 & 7.2 & 7.3 & 6.5 & $0.0(0.250)$ & $0.0(0.798)$ \\
\hline Pyrazinamide $5 \times 10^{-5} \mathrm{M}$ & 7.7 & 7.7 & 7.2 & 3.5 & 5.5 & 5.5 & 5.7 & 5.2 & 3.7 & $24.5(0.503)$ & $4.8(0.949)$ \\
\hline
\end{tabular}

The effects of exposure to a range of antibiotics on the viability of Onchocerca gutturosa adult males in long-term ( 40 day) in vitro culture. Viability was assessed by measuring worm motility scores throughout the trial and by MTT colorimetry on day 40 .

(maximum) [22] at regular intervals up to 40 days. The biochemical evaluation of worm viability was carried out by MTT/formazan colorimetry on day 40 . In this assay, the yellow compound MTT [3-(4,5-dimethylthiazol-2-yl)2,5-diphenyltetrazolium bromide] is reduced by the mitochondrial enzyme succinate dehydrogenase of living tissues to produce the blue precipitate MTT formazan $[24,25]$. Single intact worms were placed in each well of a 48-well plate (Falcon, UK) containing $0.5 \mathrm{ml}$ of $0.5 \mathrm{mg} /$ ml MTT (Sigma, UK) in phosphate buffered saline, and incubated for 30 minutes at $37^{\circ} \mathrm{C}$. The worms were then transferred to separate wells of a 96 well plate, each containing $200 \mu \mathrm{l}$ of dimethyl sulphoxide to solubilize the formazan. After one hour the plate was gently agitated to disperse the colour evenly and the absorbance value (optical density) of the resulting formazan solution was deter- mined at $490 \mathrm{~nm}$ on an ELISA reader. Inhibition of formazan formation is correlated with worm damage or death. The motility and MTT assay results were expressed as a mean percentage reduction compared with untreated control values at day 40. Comparisons of test groups to untreated controls for both motility levels and MTT colorimetry on day 40 were carried out using a 2-sample t-test.

\section{Contribution of Wolbachia to the MTT reduction assay}

Since the B. malayi and Drosophila melanogaster Wolbachia genomes contain genes for succinate dehydrogenase [30], the mosquito cell-line $\mathrm{C} 6 / 36$ was used to investigate the contribution of Wolbachia to the MTT reduction assay. C6/ 36 is derived from Aedes albopictus larvae and is not naturally infected with Wolbachia. However, it can be easily infected with Wolbachia from the cell-line Aa23, which is

Table 2: Summary of long-term trial 2: parasite mean motility scores and viability (MTT colorimetry)

\begin{tabular}{|c|c|c|c|c|c|c|c|c|c|c|c|}
\hline \multirow{2}{*}{$\begin{array}{l}\text { Compound/drug and conc. } \\
\text { DAY }\end{array}$} & \multicolumn{9}{|c|}{ Mean motility scores over a period of 40 days } & \multirow{2}{*}{$\begin{array}{l}\text { Motility } \\
\begin{array}{l}\% \text { reduction on } \\
\text { day } 40(P)\end{array}\end{array}$} & \multirow{2}{*}{$\begin{array}{c}\text { MTT } \\
\% \text { inhibition on day } \\
40(P)\end{array}$} \\
\hline & I & 5 & 10 & 15 & 20 & 25 & 30 & 35 & 40 & & \\
\hline Control & 7.6 & 7.2 & 7.1 & 6.8 & 5.9 & 4.9 & 5.8 & 6.1 & 6.3 & & \\
\hline aCGP 6I40 I.25 × 10-5M (positive control) & 1.3 & 0.0 & 0.0 & 0.0 & 0.0 & 0.0 & 0.0 & 0.0 & 0.0 & 100.0 (ND) & 87.2 (ND) \\
\hline bNorfloxacin $5 \times 10^{-5} \mathrm{M}$ & 6.9 & 7.4 & 7.1 & 2.4 & 2.5 & 2.6 & 1.4 & 1.0 & 2.5 & $60.3(\mathrm{ND})$ & 90.7 (ND) \\
\hline Ciprofloxacin $5 \times 10^{-5} \mathrm{M}$ & 6.9 & 7.9 & 7.1 & 3.6 & 4.1 & 3.0 & 2.3 & 2.5 & 3.0 & $52.4(0.080)$ & $73.3(0.040)$ \\
\hline Vancomycin $5 \times 10^{-5} \mathrm{M}$ & 6.4 & 7.4 & 6.5 & 3.6 & 3.6 & 2.8 & 2.4 & 1.6 & 2.1 & $66.7(0.009)$ & $83.7(0.022)$ \\
\hline Gentamycin $5 \times 10^{-5} \mathrm{M}$ & 7.6 & 5.5 & 6.3 & 2.8 & 2.6 & 0.8 & 1.5 & 1.0 & 1.6 & $74.6(0.005)$ & $83.7(0.017)$ \\
\hline Ceftriaxone $5 \times 10^{-5} \mathrm{M}$ & 7.1 & 6.8 & 7.0 & 2.4 & 2.0 & 0.9 & 0.6 & 0.6 & 0.7 & $88.9(<0.001)$ & $90.7(0.011)$ \\
\hline cTriclosan $5 \times 10-5 \mathrm{M}$ & 6.9 & 4.9 & 0.4 & 0.1 & 0.0 & 0.0 & 0.0 & 0.0 & 0.0 & $100.0(N D)$ & $98.8(\mathrm{ND})$ \\
\hline${ }^{c}$ Cerulenin $5 \times 10^{-5} \mathrm{M}$ & 0.0 & 0.0 & 0.0 & 0.0 & 0.0 & 0.0 & 0.0 & 0.0 & 0.0 & 100.0 (ND) & 100.0 (ND) \\
\hline
\end{tabular}

a. Day 40 results based on I worm; 5 worms lost to microbial contamination

b. Day 40 results based on 2 worms; 4 worms lost to microbial contamination

c. Denotes compounds that were toxic to the monkey kidney cell feeder layer

The effects of exposure to a range of antibiotics on the viability of Onchocerca gutturosa adult males in long-term (40 day) in vitro culture. Viability was assessed by measuring worm motility scores throughout the trial and by MTT colorimetry on day 40 . 
Table 3: Summary of long-term trial 3: parasite mean motility scores and viability (MTT colorimetry)

\begin{tabular}{|c|c|c|c|c|c|c|c|c|c|c|c|}
\hline \multirow{2}{*}{$\begin{array}{l}\text { Compound/drug and conc. } \\
\text { DAY }\end{array}$} & \multicolumn{9}{|c|}{ Mean motility scores over a period of $\mathbf{4 0}$ days } & \multirow{2}{*}{$\begin{array}{l}\text { Motility } \\
\begin{array}{l}\text { reduction on } \\
\text { day } 40(P)\end{array}\end{array}$} & \multirow{2}{*}{$\begin{array}{c}\text { MTT } \\
\begin{array}{c}\text { inhibition on } \\
\text { day } 40(P)\end{array}\end{array}$} \\
\hline & 1 & 5 & 10 & 15 & 20 & 25 & 30 & 35 & 40 & & \\
\hline Control & 8.4 & 8.3 & 7.6 & 7.5 & 7.8 & 7.7 & 7.6 & 7.2 & 7.2 & & \\
\hline CGP $61401.25 \times 10^{-5} \mathrm{M}$ (positive control) & 2.5 & 0.3 & 0.0 & 0.0 & 0.0 & 0.0 & 0.0 & 0.0 & 0.0 & $100.0(<0.001)$ & $67.4(0.0032)$ \\
\hline Rifampicin $5 \times 10^{-5} \mathrm{M}$ & 8.0 & 6.6 & 2.1 & 0.4 & 0.4 & 0.0 & 0.0 & 0.0 & 0.0 & $100.0(<0.001)$ & $67.4(<0.001)$ \\
\hline Erythromycin $5 \times 10-5 \mathrm{M}$ & 8.4 & 8.5 & 8.0 & 7.6 & 7.7 & 7.6 & 7.4 & 7.5 & 7.4 & $0.0(0.539)$ & $0.0(0.210)$ \\
\hline Oxytetracycline $5 \times 10^{-5} \mathrm{M}$ & 8.0 & 8.1 & 7.7 & 7.5 & 7.6 & 7.5 & 7.1 & 6.4 & 6.9 & $4.2(0.360)$ & $0.0(0.995)$ \\
\hline Isoniazid $5 \times 10-5 \mathrm{M}$ & 8.2 & 8.2 & 7.7 & 8.0 & 7.7 & 7.3 & 6.8 & 5.7 & 5.2 & $27.8(0.010)$ & $35.5(0.074)$ \\
\hline Isoniazid $5 \times 10^{-5} \mathrm{M}+$ Rifampicin $5 \times 10^{-5} \mathrm{M}$ & 7.8 & 8.2 & 5.0 & 4.0 & 2.7 & 0.8 & 0.0 & 0.0 & 0.0 & $100.0(<0.001)$ & $71.7(0.002)$ \\
\hline Trimethoprim $5 \times 10^{-5} \mathrm{M}$ & 8.4 & 8.4 & 7.9 & 7.6 & 7.6 & 8.0 & 7.5 & 7.0 & 7.0 & $2.8(0.549)$ & $0.0(0.319)$ \\
\hline Sulphamethoxazole $5 \times 10^{-5} \mathrm{M}$ & 8.1 & 8.3 & 8.4 & 7.6 & 7.6 & 8.0 & 7.5 & 7.3 & 7.1 & $\mathrm{I} .4(0.848)$ & $0.0(0.998)$ \\
\hline Trimeth. $5 \times 10^{-5} \mathrm{M}+$ Sulphamethox. $5 \times 10^{-5} \mathrm{M}$ & 8.5 & 8.4 & 8.0 & 7.5 & 6.8 & 7.5 & 7.1 & 7.4 & 6.9 & $4.2(0.449)$ & $0.0(0.828)$ \\
\hline Clofazimine $1.25 \times 10-5 \mathrm{M}$ & 7.7 & 6.5 & 5.2 & 3.5 & 4.3 & 3.5 & 3.2 & 0.8 & 0.0 & $100.0(<0.001)$ & $77.4(<0.001)$ \\
\hline Clofazimine $3.13 \times 10^{-6 \mathrm{M}}$ & 8.2 & 7.0 & 4.5 & 5.5 & 5.0 & 4.8 & 3.4 & 1.2 & 0.8 & $88.9(<0.001)$ & $53.3(0.028)$ \\
\hline
\end{tabular}

The effects of exposure to a range of antibiotics and antibiotic combinations on the viability of Onchocerca gutturosa adult males in long-term ( 40 day) in vitro culture. Viability was assessed by measuring worm motility scores throughout the trial and by MTT colorimetry on day 40.

derived from A. albopictus eggs and is naturally infected with Wolbachia pipientis [31]. The viability of neither cell is affected by either the presence or absence of the bacteria, since, unlike nematodes, insects do not have a mutualistic association with their Wolbachia. To infect C6/36 cells, medium from a confluent culture of Aa23 cells was collected, filtered through a $0.8 \mu \mathrm{m}$ syringe filter to remove any whole mosquito cells, and placed into a flask of confluent C6/36 cells from which the medium had been removed. The flask was agitated gently at room temperature for 30 minutes before being incubated at $37^{\circ} \mathrm{C}$ in an atmosphere of $5 \% \mathrm{CO}_{2}$ in air. Infection of the cells with the bacteria was confirmed by IFAT [17]. Hereafter the infected cells will be termed C6/36 Wp. C6/36 and C6/36 Wp were grown in L-15 Leibovitz medium (Gibco) supplemented with $2 \mathrm{mM}$ L-glutamine, $1 \%$ non-essential amino acids, $2 \%$ tryptose phosphate broth and 5\% foetal calf serum.

MTT reduction was compared with C6/36 and C6/36 Wp cells each with and without tetracycline treatment $(20 \mu \mathrm{g} /$ $\mathrm{ml}$ for up to four weeks, with medium changed every 3 days), with at least four repeats of 250000 cells in each group. The media were removed and the cells washed and pelleted, then MTT was added to give a final concentration of $0.5 \mathrm{mg} / \mathrm{ml}$ in phosphate buffered saline. After an incubation of 1 hour at $37^{\circ} \mathrm{C}$ with $5 \% \mathrm{CO}_{2}$, the cells were washed and pelleted, and $200 \mu$ l dimethyl sulphoxide was added to release the blue formazan reduction product. The optical density of the samples was measured at 490 $\mathrm{nm}$ and the results expressed as mean optical density $( \pm$ S.D.). Student's $t$-test was used to compare differences between two groups, with $P$ values of $<0.05$ considered to be significant.

\section{Results}

\section{In vitro drug screen}

The motility scores and percentage inhibitions of MTT reduction by all the tested compounds are shown in Tables 1, 2 and 3. The positive control, CGP 6140, had a very rapid onset of action, with reductions in worm motility even on the first day after initiation of treatment. No worm movement was observed after day 15 , and by day 40 there was a 67.4 to 83.3 inhibition in formazan formation compared to untreated controls. In contrast, the average motility score of the untreated control worms in each experiment ranged from 4.9 to 7.2 at day 40 (Tables 1,2 and 3 ).

Several anti-mycobacterial drugs were tested. Of these, rifampicin $\left(5 \times 10^{-5} \mathrm{M}\right)$ was the most effective, completely inhibiting motility by day 40 (Table 1 and Fig. 1) or day 25 (Table 3). Clofazimine $\left(1.25 \times 10^{-5} \mathrm{M}\right.$ and $3.13 \times 10^{-}$ ${ }^{6} \mathrm{M}$ ) gradually affected worm motility so that, by day 40 , there were $100 \%$ and $88.9 \%$ reductions in motility, and 77.4 and 53.3 inhibition of MTT reduction, with the higher and lower concentrations, respectively (Table 3 and Fig. 4). Ethambutol $\left(5 \times 10^{-5} \mathrm{M}\right)$ was less effective, giving $44.9 \%$ and $73 \%$ reductions in motility and MTT formazan formation, respectively (Table 1 and Fig. 5). However, the other agents with activity against Mycobacterium species showed limited (pyrazinamide, isoniazid) or no (dapsone) activity against the worms (Tables 1 and 3 ). Also, the addition of isoniazid $\left(5 \times 10^{-5} \mathrm{M}\right)$ to rifampicin $\left(5 \times 10^{-5} \mathrm{M}\right)$ did not improve the efficacy of the latter (Table 3).

Doxycycline was effective (100\% reduction in motility by day $25,93 \%$ inhibition of formazan formation at day 40 ) at a concentration of $5 \times 10-5 \mathrm{M}$, but showed no activity at 


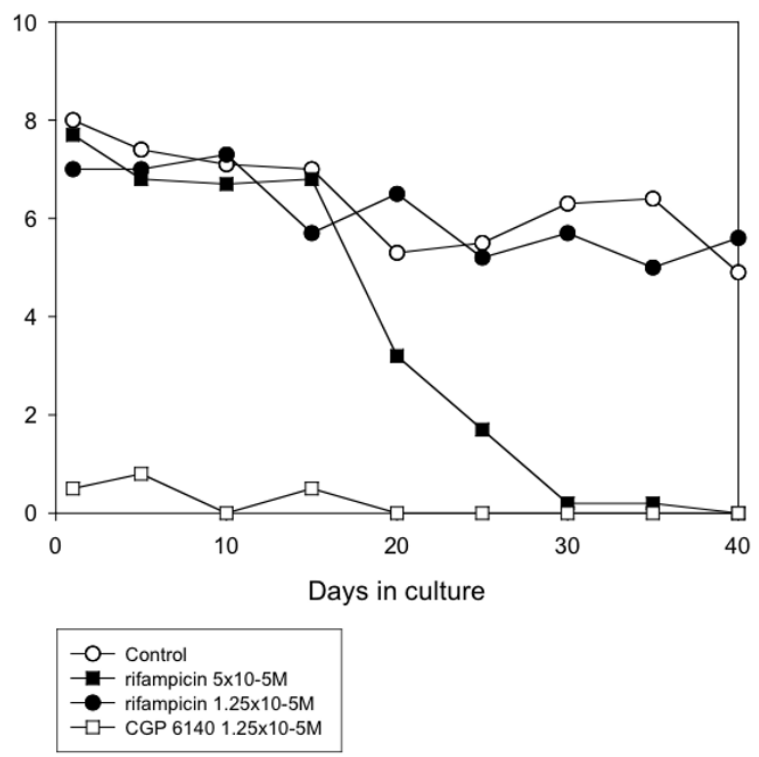

Figure I

Mean motility score of $O$. gutturosa adult males in vitro exposed to rifampicin. Control, $\bigcirc$, rifampicin $5 \times$ $10^{-5} \mathrm{M}$, rifampicin I.25 × 10-5 M, O; CGP 6I40 I.25 × 10$5 \mathrm{M}, \square$ (positive control). (Data from Table I).

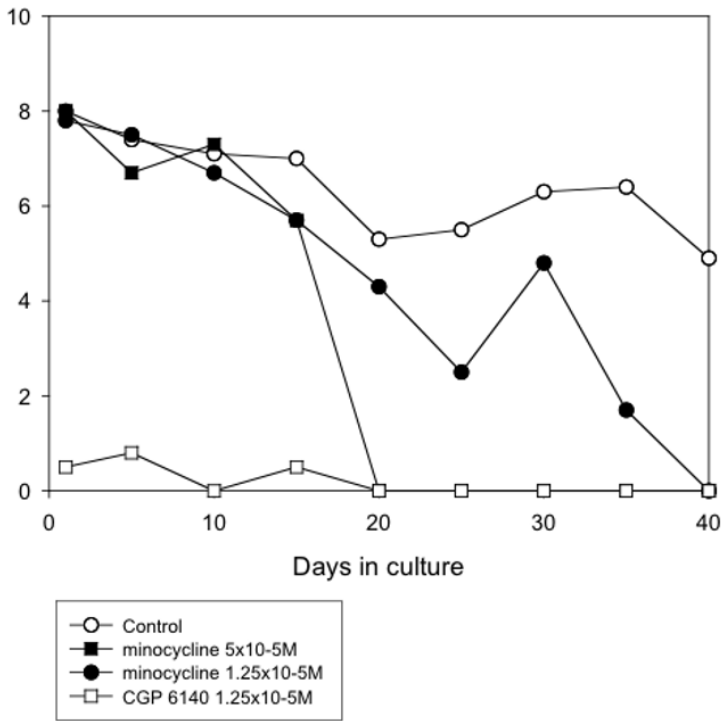

Figure 2

Mean motility score of 0 . gutturosa adult males in vitro exposed to minocycline. Control, $\bigcirc$; minocycline 5

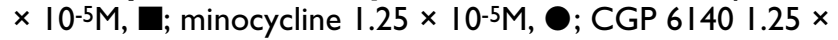
I0-5 M, $\square$ (positive control). (Data from Table I).

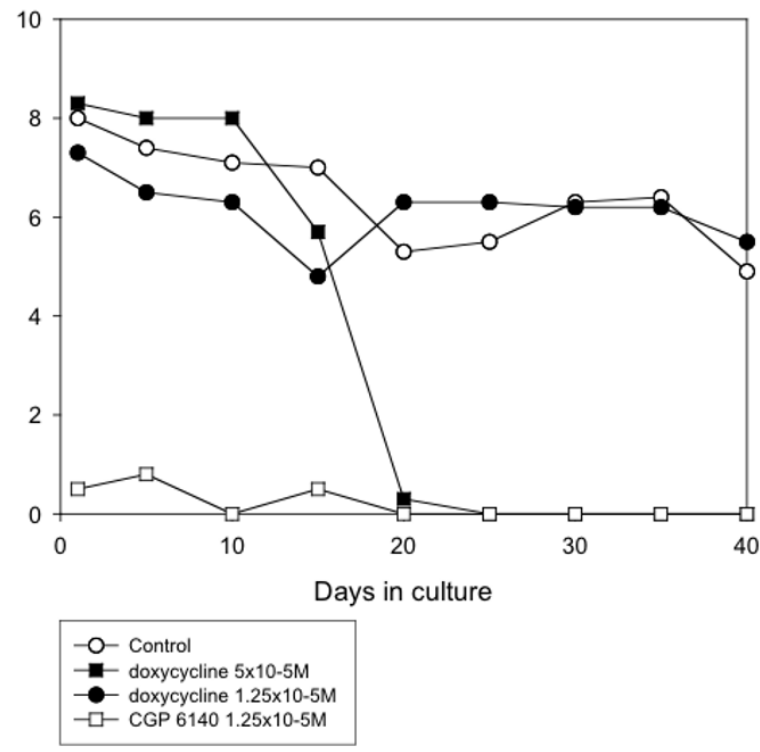

Figure 3

Mean motility score of 0 . gutturosa adult males in vitro exposed to doxycycline. Control, $\bigcirc$, doxycycline 5 $\times 10^{-5} \mathrm{M}$, 口; doxycycline I.25 × 10-5 M, O; CGP 6I40 I.25 × 10-5M, $\square$ (positive control). (Data from Table I).

$1.25 \times 10-5 \mathrm{M}$ (Fig. 3). However, minocycline was filaricidal at both of these concentrations (Fig. 2). Intermediate activity was shown by norfloxacin, ciprofloxacin, vancomycin and gentamycin (all at a concentration of $5 \times 10$ $5 \mathrm{M})$. The drugs that did not have a filaricidal effect at $5 \times$ $10-5 \mathrm{M}$ included erythromycin, oxytetracycline, trimethoprim and sulphamethoxazole (these two either alone or in combination).

Two compounds, triclosan and cerulenin, were toxic to the monkey kidney cell feeder layer, so it was not possible to conclude if they had an anti-Onchocerca effect, since a viable cell layer is essential to the long-term survival of the worms.

\section{Contribution of Wolbachia to the MTT reduction assay}

C6/36 cells with and without $W$. pipientis infection were incubated in medium or medium plus tetracycline for two or four weeks before being analysed by the MTT reduction assay. This assay showed that infection with Wolbachia did contribute to extra metabolic activity in the cells, since $\mathrm{C} 6 / 36 \mathrm{Wp}$ had significantly higher absorbance readings than C6/36 $(P=0.017$ after two weeks' incubation; $P=$ 0.000 after four weeks' incubation, Fig. 6). However, in $\mathrm{C} 6 / 36 \mathrm{Wp}$ treated with tetracycline for two to four weeks the absorbance was no different from C6/36 with tetracycline ( $P=0.269$ at two weeks; $P=0.475$ at four weeks). At neither time-point did uninfected $\mathrm{C} 6 / 36$ treated with tet- 


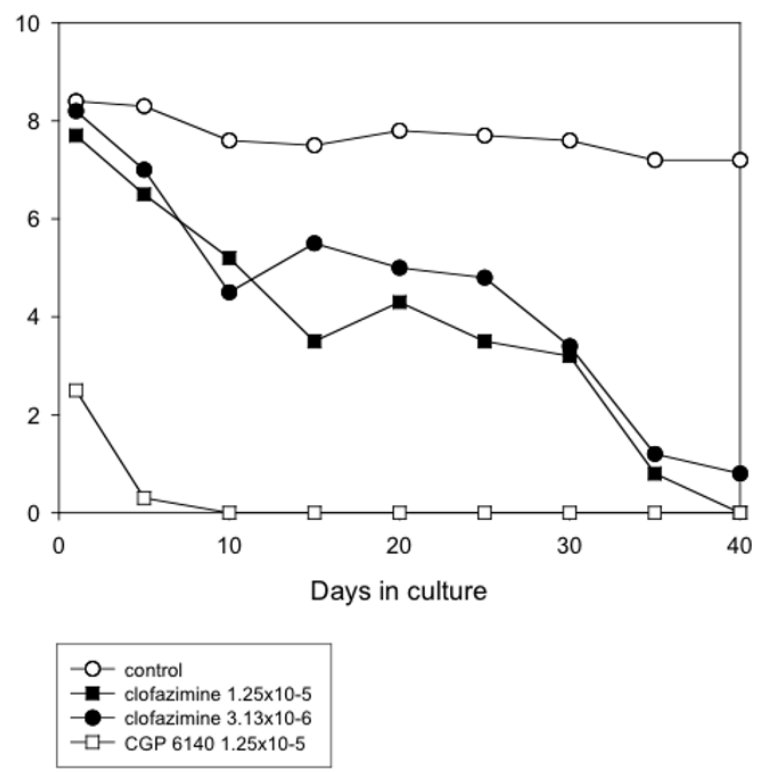

Figure 4

Mean motility score of 0 . gutturosa adult males in vitro exposed to clofazimine. Control, $\bigcirc$; clofazimine $1.25 \times 10^{-5} \mathrm{M}, \mathbf{\square}$; clofazimine $3.13 \times 10-6 \mathrm{M}, 0$; CGP 6140 I.25 × I0-5M, $\square$ (positive control). (Data from Table 3).

racycline show a significant difference from those not treated $(P=0.958$ at two weeks; $P=0.543$ at four weeks, Fig. 6), indicating that the tetracycline itself had no effect in reducing the metabolic activity.

\section{Discussion}

The results presented here confirm that O.gutturosa males provide a suitable in vitro screen for slow-acting antibiotic drugs with macrofilaricidal activity. Male O.gutturosa are smaller than O.volvulus males but contain an almost identical Wolbachia/nematode ratio (H.F. McGarry and M.J. Taylor, unpublished observation) indicating that they are a suitable model for human onchocerciasis.

The gradual reduction in motility (as with clofazimine) or a delay before effects were seen (rifampcin, doxycycline, minocycline) is consistent with activity against the endosymbionts, in contrast to the direct and rapid effects on worm viability of the positive control, Amocarzine. Interestingly, with rifampcin, doxycycline, minocycline and ethambutol, the most rapid decline in worm motility occurred between 15 and 20 days of treatment. This period of treatment may be sufficient to inhibit Wolbachia-dependent processes, such as inhibition of protein synthesis (which is the mode of action of tetracyclines), with a resultant deterioration in nematode health before

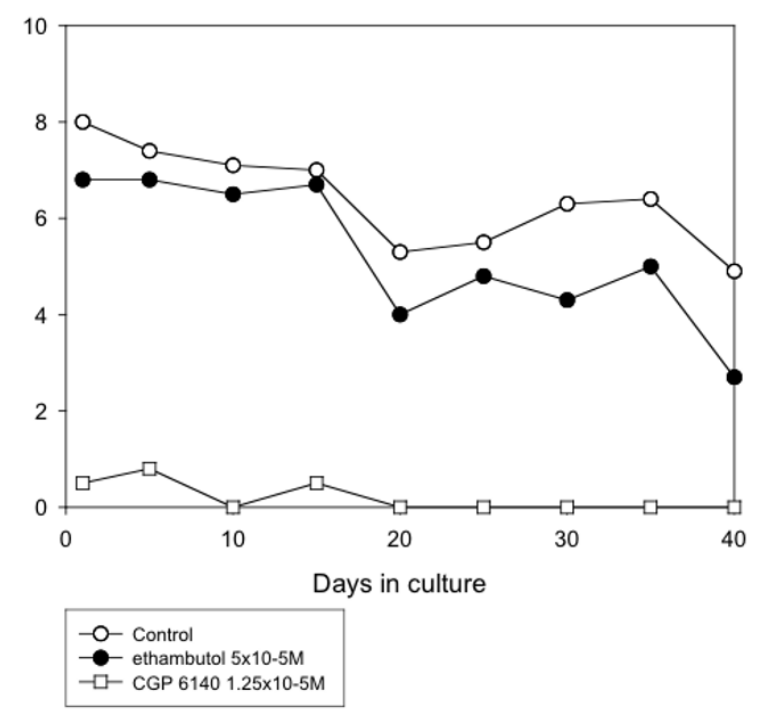

Figure 5

Mean motility score of 0 . gutturosa adult males in vitro exposed to ethambutol. Control, $\bigcirc$; ethambutol $5 \times$ I0-5M, O CGP 6I40 I.25 × 10-5 M, $\square$ (positive control). (Data from Table I).

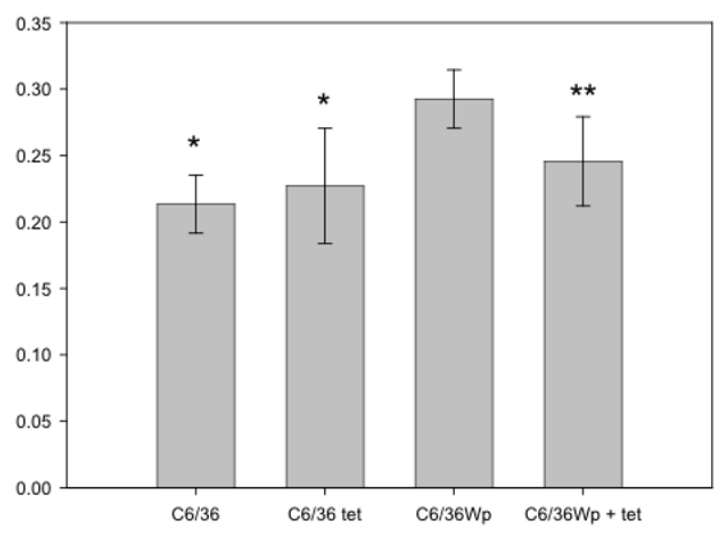

Figure 6

Wolbachia contribute to metabolic activity as measured by the MTT reduction assay $\mathrm{C} 6 / 36$ and $\mathrm{C} 6 / 36 \mathrm{Wp}$ were incubated in medium or medium with tetracycline 20 $\mu \mathrm{g} / \mathrm{ml}$ for four weeks before the MTT reduction assay was performed. Bars represent means of five repeats each of 250 000 cells $( \pm$ S.D.). Values that are significantly lower than those of $C 6 / 36 \mathrm{WP}$ are denoted by $*(P=0.000)$ and $* *(P=$ 0.03). 
a decline in bacterial numbers is observable. As such, this O. gutturosa system is likely to be more sensitive than one that utilises Wolbachia-infected mosquito cell lines, which relies on direct observation for the presence or absence of bacteria $[27,32]$.

Currently, doxycycline (200 mg) administered daily for three weeks is the shortest effective regimen that has been tried against a human filarial infection; when followed by standard anti-filarial chemotherapy, this treatment resulted in prolonged reductions in microfilaraemia and in Wolbachia numbers in the microfilariae, but was not macrofilaricidal [20] and is similar to the timeframe after which effects were observable in vitro.

Rifampicin has previously been shown to be very effective against Wolbachia $[6,27,32,33]$, which was confirmed in this in vitro antifilarial assay. Rifampicin interferes with nucleic acid synthesis by combining with and inhibiting the RNA-polymerase of bacteria and is bactericidal. The anti-leprosy agent clofazimine also demonstrated good activity against $O$. gutturosa, and is also bactericidal. Antituberculosis and -leprosy therapies may have benefits at the population level by reducing the prevalence of filariasis [34]. However, the other drugs tested that are used against Mycobacterium species (ethambutol, dapsone, pyrazinamide, isoniazid) showed little anti-Onchocerca activity.

The tetracycline doxycycline was filaricidal in this drug screen, confirming previous findings that it has anti-Wolbachia effects $[27,33,35]$. This drug has also been used in human filarial infections, in which it has resulted in a prolonged loss of microfilariae and lack of embryogenesis $[10,11,14]$, and has recently been shown to be macrofilaricidal against $W$. bancrofti [13]. In the present work minocycline was even more effective than doxycycline and warrants further investigation in vivo. This result agrees with our unpublished work (S. Townson) that minocycline is more active than doxycycline against $O$. lienalis microfilariae in mice. Minocycline has the advantage over doxycycline of inducing less phototoxicity. However, oxytetracycline showed no anti-Wolbachia activity, in contrast to previous reports $[6,9,32]$; it is not clear why there were contradictory findings. The lack of activity by other compounds (erythromycin, trimethoprim and sulphamethoxazole) was consistent with previous findings $[27,36]$.

Filarial nematodes cultured in vitro often succumb to drug treatments more readily than they do in vivo. The system described here provides a relatively easy and inexpensive way to perform a primary or secondary screen of compounds for anti-filarial/Wolbachia activity, which would then be followed by in vivo experiments. Antibiotics are slow-acting against filarial nematodes, as seen in the present study and in field trials (for example, [12,13]); an advantage of the male $O$. gutturosa culture system is that it can be maintained for at least 40 days. In the present study the effects of the compounds has not been directly related to their activity against Wolbachia and it is possible that they also had direct anti-nematodal activity. Studies on the dynamics of the loss of the bacteria from worms are currently underway using quantitative polymerase chain reaction and/or immunohistology in the screening system.

The presence of Wolbachia made a significant contribution to the MTT assay in C6/36 cells as would be predicted from the presence of succinate dehydrogenase as determined by genomic annotation. Thus partial reduction in formazan formation in MTT assays could reflect either the loss of bacteria and retention of worm viability or vice versa. Should an in vitro screen for specific activity against either the bacteria or worm be required then alternative markers of viability may need to be developed [37].

\section{Conclusion}

This study has demonstrated that the in vitro screen for macrofilaricidal activity, which uses the culture of male $O$. gutturosa, can be successfully extended and is also valid for the screening of antibiotic compounds with potential anti-Wolbachia/filarial activity. This system can be used for long-term screening, in this case for 40 days. Rifampicin and doxycycline were two of the most active antibiotics tested in this screen, in agreement with previous findings. However, a new finding was that minocycline was more quickly and completely effective than either of these compounds. It was also found that Wolbachia contribute to the MTT formazan formation which is used as a marker of filarial worm viability, suggesting that bacteria contribute directly to the metabolic activity of the nematode and that it may be necessary to reassess alternative indicators of worm and or bacterial viability.

\section{Abbreviations}

MTT, 3-(4,5-dimethylthiazol-2-yl)-2,5-diphenyltetrazolium bromide; MDA, mass drug administration.

\section{Competing interests}

The author(s) declare that they have no competing interests.

\section{Authors' contributions}

STo designed the antibiotic screen experiments, analysed the results and advised on the manuscript preparation. STo and STa performed the antibiotic screen experiments. HFM analysed results, performed statistical analysis and prepared the manuscript. GLE performed the C6/36 MTT analyses. MJT designed the C6/36 MTT assay experiments, 
analysed the results and advised on the manuscript preparation.

\section{Acknowledgements}

This investigation received financial assistance from UNICEF/UNDP/World Bank/WHO Special Programme for Research and Training in Tropical Diseases (TDR). MJT is funded by a Senior Fellowship in Basic Biomedical Science awarded by the Wellcome Trust.

\section{References}

I. Ardelli BF, Guerriero SB, Prichard RK: Genomic organization and effects of ivermectin selection on Onchocerca volvulus P-glycoprotein. Molecular and Biochemical Parasitology 2005, 143:58-66.

2. Eng JKL, Prichard RK: A comparison of genetic polymorphism in populations of Onchocerca volvulus from untreated- and ivermectin-treated patients. Molecular and Biochemical Parasitology 2005, 142:193-202.

3. Schwab A, Boakye D, Kyelem D, Prichard RK: Detection of benzimidazole resistance-associated mutations in the filarial nematode Wuchereria bancrofti and evidence for selection by albendazole and ivermectin combination treatment. American Journal of Tropical Medicine and Hygiene 2005, 73:234-238.

4. Taylor MJ, Bandi C, Hoerauf A: Wolbachia bacterial endosymbionts of filarial nematodes. Advances in Parasitology 2005, 60:245-284.

5. Hoerauf $A$, Nissen-Pähle $K$, Schmetz $C$, Henkle-Dührsen $K$, Blaxter ML, Büttner DW, Gallin MY, Al-Qaoud KM, Lucius R, Fleischer B: Tetracycline therapy targets intracellular bacteria in the filarial nematode Litomosoides sigmodontis and results in filarial infertility. Journal of Clinical Investigation 1999, 103: I I-18.

6. Townson S, Hutton D, Siemienska J, Hollick L, Scanlon T, Tagboto SK, Taylor MJ: Antibiotics and Wolbachia in filarial nematodes: antifilarial activity of rifampicin, oxytetracycline and chloramphenicol against Onchocerca gutturosa, Onchocerca lienalis and Brugia pahangi. Annals of Tropical Medicine and Parasitology 2000, 94:80|-816.

7. Chirgwin SR, Coleman SU, Porthouse KH, Nowling JM, Punkosdy GA Klei TR: Removal of Wolbachia from Brugia pahangi is closely linked to worm death and fecundity but does not result in altered lymphatic lesion formation in Mongolian gerbils (Meriones unguiculatus). Infection and Immunity 2003, 7I:6986-6994.

8. Chirgwin SR, Nowling JM, Coleman SU, Klei TR: Brugia pahangi and Wolbachia: the kinetics of bacteria elimination, worm viability, and host responses following tetracycline treatment. Experimental Parasitology 2003, 103:16-26.

9. Langworthy NG, Renz A, Mackenstedt U, Henkle-Dührsen K, de Bronsvoort MB, Tanya VN, Donnelly MJ, Trees AJ: Macrofilaricidal activity of tetracycline against the filarial nematode Onchocerca ochengi : elimination of Wolbachia precedes worm death and suggests a dependent relationship. Proceedings of the Royal Society of London Series B-Biological Sciences 2000 267:1063-1069.

10. Hoerauf A, Volkmann L, Hamelmann C, Adjei O, Autenrieth IB, Fleischer B, Büttner DW: Endosymbiotic bacteria in worms as targets for a novel chemotherapy in filariasis. Lancet 2000 , 355: $1242-1243$.

II. Hoerauf A, Mand S, Adjei O, Fleischer B, Büttner DW: Depletion of wolbachia endobacteria in Onchocerca volvulus by doxycycline and microfilaridermia after ivermectin treatment. Lancet 200I, 357:|4|5-|4|6.

12. Hoerauf A, Mand S, Volkmann L, Büttner M, Marfo-Debrekyei Y, Taylor M, Adjei O, Büttner DW: Doxycycline in the treatment of human onchocerciasis: kinetics of Wolbachia endobacteria reduction and of inhibition of embryogenesis in female Onchocerca worms. Microbes and Infection 2003, 5:26I-273.

13. Taylor MJ, Makunde WH, McGarry HF, Turner JD, Mand S, Hoerauf A: Macrofilaricidal activity after doxycycline treatment of Wuchereria bancrofti : a double-blind, randomised placebocontrolled trial. Lancet 2005, 365:2I|6-2I2I.

14. Hoerauf A, Mand S, Fischer K, Kruppa T, Marfo-Debrekyei Y, Yaw Debrah A, Pfarr KM, Adjei O, Büttner DW: Doxycycline as a novel strategy against bancroftian filariasis - depletion of Wol- bachia endosymbionts from Wuchereria bancrofti and stop of microfilaria production. Medical Microbiology and Immunology 2003, 192:211-216

15. Taylor MJ, Cross HF, Bilo K: Inflammatory responses induced by the filarial nematode Brugia malayi are mediated by an LPSlike substance from endosymbiotic Wolbachia bacteria. Journal of Experimental Medicine 2000, 8: | 429-1 435.

16. Saint André AV, Blackwell NM, Hall LR, Hoerauf A, Brattig NW, Volkmann L, Taylor MJ, Ford L, Hise AG, Lass JH, Diaconu E, Pearlman E: The role of endosymbiotic Wolbachia bacteria in the pathogenesis of river blindness. Science 2002, 295: I892-1895

17. Gillette-Ferguson I, Hise AG, McGarry HF, Turner J, Esposito A, Sun $\mathrm{Y}$, Diaconu E, Taylor MJ, Pearlman E: Wolbachia-induced neutrophil activation in a mouse model of ocular onchocerciasis (river blindness). Infection and Immunity 2004, 72:5687-5692.

18. Cross HF, Haarbrink M, Egerton G, Yazdanbakhsh M, Taylor MJ: Severe adverse reactions to filarial chemotherapy are associated with the release of Wolbachia endosymbionts into blood. Lancet 200I, 358:1873-1875.

19. Keiser PB, Reynolds SM, Awadzi K, Ottesen EA, Taylor MJ, Nutman TB: Bacterial endosymbionts of Onchocerca volvulus in the pathogenesis of posttreatment reactions. Journal of Infectious Diseases 2002, |85:805-8|I.

20. Turner JD, Mand S, Yaw Debrah A, Muehlfeld J, Pfarr K, McGarry HF, Adjei O, Taylor MJ, Hoerauf A: A randomised, double blind clinical trial of three week doxycycline plus albendazole and ivermectin in the treatment of Wuchereria bancrofti. Clinical Infectious Diseases 2006, 42:108I-1089.

21. Townson S: The development of a laboratory model for onchocerciasis using Onchocerca gutturosa : in vitro culture, collagenase effects, drug studies and cryopreservation. Tropical Medicine and Parasitology 1988, 39:475-479.

22. Townson S, Connelly C, Dobinson A, Muller R: Drug activity against Onchocerca gutturosa males in vitro: a model for chemotherapeutic research on onchocerciasis. Journal of Helminthology 1987, 61:27I-281.

23. Townson S, Tagboto SK: Onchocerca gutturosa : sensitivity to a range of antibiotic and antiparasitic drugs. In The I8th International Conference of the World Association for the Advancement of Veterinary Parasitology Italy. Abs: I58.

24. Comley JCW, Rees MJ, Turner CH, Jenkins DC: Colorimetric quantitation of filarial viability. International Journal for Parasitology 1989, 19:77-83.

25. Comley JCW, Townson S, Rees MJ, Dobinson A: The further application of MTT-formazan colorimetry to studies on filarial viability. Tropical Medicine and Parasitology 1989, 40:3।I-316.

26. Townson S, Connelly C, Muller R: Optimization of culture conditions for the maintenance of Onchocerca gutturosa adult worms in vitro. Journal of Helminthology 1986, 60:323-330.

27. Fenollar F, Maurin M, Raoult D: Wolbachia pipientis growth kinetics and susceptibilities to 13 antibiotics determined by immunofluorescence staining and real-time PCR. Antimicrobial Agents and Chemotherapy 2003, 47:1665-167I.

28. Poltera AA, Zea-Flores G, Guderian R, Beltranena F, Proana R, Moran $M$, Zak F, Striebel HP: Onchocercacidal effects of amocarzine (CGP 6 I 40) in Latin America. Lancet 1991, 337:583-584.

29. Kohler P, Davies KP, Zahner H: Activity, mechanism of action and pharmacokinetics of 2-tert-butylbenzothiazole and CGP 6140 (amocarzine) antifilarial drugs. Acta Tropica 1992, 5I:195-2II.

30. Wolbachia Genome Project: Sequencing the Brugia malayi endosymbiont [http://tools.neb.com/wolbachia/wgspindex.html]

31. O'Neill SL, Pettigrew MM, Sinkins SP, Braig HR, Andreadis TG, Tesh RB: In vitro cultivation of Wolbachia pipientis in an Aedes albopictus cell line. Insect Molecular Biology 1997, 6:33-39.

32. Hermans PG, Hart CA, Trees AJ: In vitro activity of antimicrobial agents against the endosymbiont Wolbachia pipientis. Journal of Antimicrobial Chemotherapy 200I, 47:659-663.

33. Volkmann L, Fischer K, Taylor M, Hoerauf A: Antibiotic therapy in murine filariasis (Litomosoides sigmodontis): comparative effects of doxycycline and rifampicin on Wolbachia and filarial viability. Tropical Medicine \& International Health 2003, 8:392-40I.

34. Sherchand J, Obsomer V, Das Thakur G, Hommel M: Mapping of lymphatic filariasis in Nepal. Filaria Journal 2003, 2: 
35. Rao R, Weil GJ: In vitro effects of antibiotics on Brugia malayi worm survival and reproduction. Journal of Parasitology 2002, 88:605-6II.

36. Hoerauf A, Volkmann L, Nissen-Paehle K, Schmetz C, Autenrieth I, Büttner DW, Fleischer B: Targeting of Wolbachia endobacteria in Litomosoides sigmodontis: comparison of tetracyclines with chloramphenicol, macrolides and ciprofloxacin. Tropical Medicine \& International Health 2000, 5:275-279.

37. Comley JCW, Rees MJ, O'Dowd A: The application of biochemical criteria to the assessment of macrofilarial viability. Tropical Medicine and Parasitology 1988, 39:456-459.

Publish with Bio Med Central and every scientist can read your work free of charge

"BioMed Central will be the most significant development for disseminating the results of biomedical research in our lifetime. "

Sir Paul Nurse, Cancer Research UK

Your research papers will be:

- available free of charge to the entire biomedical community

- peer reviewed and published immediately upon acceptance

- cited in PubMed and archived on PubMed Central

- yours - you keep the copyright

Submit your manuscript here:

http://www.biomedcentral.com/info/publishing_adv.asp 